\title{
Evaluating the association of serum ferritin and hepatic iron with disease severity in non-alcoholic fatty liver disease
}

\author{
Elena Buzzetti $^{1,2}$ (I) | Salvatore Petta ${ }^{3}$ (D) | Roberta Manuguerra ${ }^{4}$ (i) | Tu Vinh Luong ${ }^{4}$ (I) | \\ Daniela Cabibi $^{5}$ (D) | Elena Corradini ${ }^{1}$ (D) | Antonio Craxi ${ }^{3}$ (D) | Massimo Pinzani ${ }^{2}$ (D) | \\ Emmanuel Tsochatzis ${ }^{2}$ (D) | Antonello Pietrangelo ${ }^{1}$
}

${ }^{1}$ Division of Internal Medicine 2 and Center for Hemochromatosis, University of Modena and Reggio Emilia, Modena, Italy

${ }^{2} \mathrm{UCL}$ Institute for Liver and Digestive Health, Royal Free Hospital, London, UK

${ }^{3}$ Section of Gastroenterology and Hepatology, Di.Bi.M.I.S., University of

Palermo, Palermo, Italy

${ }^{4}$ Department of Cellular Pathology, Royal Free Hospital, London, UK

${ }^{5}$ Department of Sciences for the Promotion of Health and Mother and Child Care,

Anatomic Pathology, University of Palermo, Palermo, Italy

\section{Correspondence}

Elena Buzzetti, Division of Internal Medicine 2 and Center for Hemochromatosis,

University Hospital 'Policlinico', Modena, Italy.

Email: buzzetti.elena@gmail.com

Funding information

Fondazione Telethon, Grant/Award Number: GGP14285; AIGO-M.I.M.I. (Associazione

Italiana Gastroenterologi Ospedalieri

- Montecatini Interactive Medicine

International)

Handling Editor: Isabelle Leclercq

\begin{abstract}
Background \& Aims: Hyperferritinemia, with or without increased hepatic iron, represents a common finding in non-alcoholic fatty liver disease (NAFLD). However, it is unclear whether it reflects hepatic inflammation or true iron-overload and, in case the latter is confirmed, whether this influences disease progression. We therefore explored the association between serum ferritin, degree and pattern of hepatic iron deposition and liver disease severity in patients with NAFLD.

Methods: We selected 468 patients with biopsy-proven NAFLD from 2 European centres. Iron, hepatic and metabolic parameters were collected the time of liver biopsy. Iron deposits in hepatocytes and reticuloendothelial cells were assessed and graded. Diagnosis of non-alcoholic steatohepatitis (NASH) and fibrosis staging were performed.

Results: A total of 122 (26\%) patients had hyperferritinemia, whereas stainable hepatic iron was found in 116 (25\%) patients (38\% predominantly in hepatocytes, 20\% in reticuloendothelial cells and $42 \%$ in both). Subjects with stainable hepatic iron, particularly those with a mixed pattern, had higher serum ferritin and transaminases but only a mixed pattern of iron deposition was among the variables significantly associated with presence of NASH. Serum ferritin was not associated with presence of $\mathrm{NASH}$, however it increased with worsening fibrosis stage (F3 compared to F0-F1), and significantly decreased in stage F4.

Conclusions: A mixed pattern of hepatic iron deposition is associated with the presence of steatohepatitis, while serum ferritin increases with worsening fibrosis up to pre-cirrhotic stage. In individual NAFLD patients, serum ferritin could be evaluated as part of non-invasive diagnostic panels but not on its own.
\end{abstract}

Abbreviations: ALT, alanine aminotransferases; AST, aspartate aminotransferases; BMI, body mass index; CRN, clinical research network; CRP, C reactive protein; DM, type 2 diabetes mellitus; GGT, gamma-glutamiltranspeptidase; GLC, glucose; HbA1c, glycosylated hemoglobin; HCC, hepatocellular carcinoma; HC, hepatocellular; HDL, high-density lipoprotein cholesterol; HOMA, homeostatic model assessment index; IFG, impaired fasting glucose; IQR, interquartile range; LDL, low-density lipoprotein cholesterol; MetS, metabolic syndrome; NAFLD, non-alcoholic fatty liver disease; NASH, non-alcoholic steatohepatitis; NAS, NASH activity score; OR, odds ratio; PLT, platelet count; RES, reticuloendothelial; SD, standard deviation; SF, serum ferritin; TG, triglycerides; TIBC, total iron binding capacity; TSAT, transferrin saturation. 


\section{1 | INTRODUCTION}

Non-alcoholic fatty liver disease (NAFLD) is increasingly prevalent in Western countries, affecting approximately $30 \%$ of unselected population. ${ }^{1}$ It is usually diagnosed in patients with metabolic syndrome (MetS), but also in a small percentage of subjects with normal weight (7\%). ${ }^{2}$ NAFLD encompasses a wide spectrum of histological conditions, from simple steatosis to steatohepatitis or NASH (characterized by ballooning and lobular inflammation) with subsequent development of fibrosis that can lead to cirrhosis. Despite its high prevalence, only a small proportion of subjects with NAFLD develops NASH and faces a higher risk of liver disease progression. ${ }^{3}$

A high serum ferritin (SF) is a common finding in NAFLD, involving up to $30 \%$ of affected subjects, and it is often the biochemical abnormality that leads to medical attention. However, it is still unclear whether it simply reflects hepatic inflammation or represents true hepatic iron-overload. ${ }^{4,5}$ In this context, SF has been proposed as a marker of both NASH and liver fibrosis with contrasting results ${ }^{6-8}$ and has been incorporated in panels for liver fibrosis assessment. ${ }^{9}$ More recently, SF has been proposed as an independent predictor of long-term mortality in NAFLD. ${ }^{10}$

Mild-to-moderate hepatic iron accumulation is encountered in a number of liver diseases including chronic hepatitis $\mathrm{C}$, alcoholic and non-alcoholic fatty liver disease and cirrhosis, ${ }^{11}$ but the underlying mechanism is unclear. Also, although it is known that iron excess can damage the liver by inducing oxidative stress and lipid peroxidation, ${ }^{12}$ in these settings the effect of iron accumulation is not completely understood.

Moreover, the relationship between SF levels, the pattern of iron deposition (hepatocellular [HC], reticuloendothelial [RES] or mixed ${ }^{13}$ and liver disease stage in NAFLD has not been elucidated.

The aim of this study was therefore to assess the presence and pattern of hepatic iron accumulation in patients with biopsy-proven NAFLD, and to examine whether such a finding is associated with more severe/progressive liver disease. Furthermore, we analysed the role of serum ferritin as predictor of liver disease severity.

\section{PATIENTS AND METHODS}

\section{1 | Patients population}

We retrospectively evaluated all consecutive outpatients (aged $\geq 18$ years) with a liver biopsy showing NAFLD, irrespective of fibrosis severity, seen at the Hepatology clinic of the Royal Free Hospital (London, United Kingdom) or Policlinico Giaccone (Palermo, Italy) in a time frame of 20 and 12 years respectively, and finally included 468 out of 477 patients. Histological and clinical criteria for NAFLD definition and inclusion in this study

\section{Key points}

There are contrasting results on the association of serum ferritin and presence or pattern of hepatic iron deposition with disease severity in people with fatty liver. Therefore, we reviewed liver biopsies of NAFLD patients in order to provide a conclusive answer to the above question. We showed that iron deposition both in hepatocytes and macrophages (mixed pattern) was associated with non-alcoholic steatohepatitis, which is the progressive form of fatty liver. Serum ferritin alone could not be used as a marker of liver scarring (fibrosis).

were: presence of steatosis in more than $5 \%$ of hepatocytes in the absence of features characteristic of other etiologies of liver disease ${ }^{14}$; alcohol intake lower than $20 / 30 \mathrm{~g}$ /day in females/ males; absence of chronic liver disease of other etiologies such as viral hepatitis, autoimmune hepatitis, primary biliary cholangitis, hereditary hemochromatosis (evidence of iron overload and relevant genetic testing showing C282Y homozygosity, C282Y/ H63D compound heterozygosity, H63D homozygosity), Wilson's disease, alpha-1-antitrypsin deficiency based on appropriate testing. We excluded causes of secondary hepatic steatosis, such as use of fatty liver-inducing drugs or previous gastro-intestinal by-pass surgery. Clinical information including presence of hypertension (blood pressure $\geq 140 / 90 \mathrm{~mm} \mathrm{Hg}$ measured in 2 different occasions and/or antihypertensive drug treatment), type 2 diabetes mellitus (DM) or impaired fasting glucose (IFG), dyslipidemia (elevated triglycerides [TG], low-density lipoprotein cholesterol [LDL-C], decreased high-density lipoprotein cholesterol [HDL-C] $]^{15}$ or use of a lipid-lowering agent) and body mass index (BMI) were collected from clinical documentation recorded within 6 months from liver biopsy.

Metabolic syndrome was diagnosed according to the National Cholesterol Education Program Adult Treatment Panel III criteria, ${ }^{16}$ when at least 3 of the followings were present: enlarged waist circumference ( $\geq 102-88 \mathrm{~cm}$, males-females), TG $\geq 1.7 \mathrm{mmol} / \mathrm{L}$ and/or medication use for elevated TG, reduced HDL cholesterol ( $<1.0-1.3 \mathrm{mmol} / \mathrm{L}$, males-females) and/or medication use for reduced $\mathrm{HDL}$ cholesterol, blood pressure $\geq 130 / 85 \mathrm{~mm} \mathrm{Hg}$ and/or medication use for hypertension, fasting glucose $\geq 5.6 \mathrm{mmol} / \mathrm{L}$ and/or medication use for elevated glucose. Biochemical parameters included platelet count (PLT), aminotransferases (ALT, AST), bilirubin, serum albumin, gamma-glutamiltranspeptidase (GGT), fasting glucose, HOMA (homeostatic model assessment) index and glycosylated hemoglobin (HbA1c) when available. 


\section{2 | Iron status}

Serum iron, SF and total iron binding capacity (TIBC) and/or transferrin saturation (TSAT) were determined using automated biochemical methods. HFE genetic test had been performed in patients with hyperferritinemia (above $200 \mu \mathrm{g} / \mathrm{L}$ in females and $300 \mu \mathrm{g} / \mathrm{L}$ in males or menopause females) and an abnormal transferrin saturation (TSAT > 45\%) and in selected patients with hyperferritinemia and normal TSAT according to the physicians' discretion, by PCR-based techniques.

\section{3 | Histological assessment}

All liver biopsy specimens were obtained by percutaneous or trans-jugular route, with a median length of $19 \mathrm{~mm}(6-58 \mathrm{~mm})$. Specific criteria for liver biopsy were: length of at least $10 \mathrm{~mm}$, comprising at least six portal tracts or less if cirrhotic or considered of sufficient quality for a diagnosis and staging by the pathologist. We included 6 biopsies $<10 \mathrm{~mm} \mathrm{(6,} \mathrm{7,} \mathrm{8,} \mathrm{9,} 9$ and $9 \mathrm{~mm}$ respectively) presenting simple steatosis since the histopathologist was satisfied with the representativity of the sample and the results of the analysis were not changed by their removal (data not shown). Liver sections were routinely stained with haematoxylin/ eosin, silver reticulin, blue aniline or Sirius red for collagen, Perls' Prussian blue for iron. Liver biopsies were centrally reviewed by a single pathologist in each centre. NAFLD lesions were scored according to the NASH Clinical Research Network (CRN) NAS scoring system. ${ }^{17} \mathrm{NASH}$ was diagnosed in the presence of the combination of any degree of hepatic steatosis, hepatocellular ballooning and lobular inflammation. ${ }^{18,19}$ Hepatic fibrosis was staged on a 5 -point scale $(0=$ absence of fibrosis, $1=$ zone 3 perisinusoidal $/$ perivenular fibrosis, 2 = zone 3 and periportal fibrosis, 3 = septal/ bridging fibrosis, $4=$ cirrhosis $^{17}$ ). Significant and advanced fibrosis was defined as stages $\geq F 2$ and $\geq F 3$, respectively. Advanced fibrosis was chosen as one of the main variables of interest based on previous studies demonstrating that it is associated with long-term clinical outcomes and increased mortality in NAFLD patients. ${ }^{20,21}$ Inter-observer agreement regarding histological evaluation by the 2 pathologists was tested on a set of 30 slides by weighted Cohen's kappa, with a resulting $\mathrm{k}$ value for fibrosis of 0.76 , meaning excellent agreement.The presence of iron was assessed both in hepatocytes and reticuloendothelial cells and the degree of liver siderosis was classified according to a modified Scheuer's system (Table $\mathrm{S}^{13,22}$ ).

\section{4 | Ethical approval}

Blood tests and liver biopsy were performed as part of the standard or routine care. Both centres had the approval from the local ethical committee to use registered parameters and liver biopsies for studies. The study was carried out in accordance with the principles of the Helsinki Declaration.

\section{5 | Statistical analysis}

Continuous data were presented as mean \pm standard deviation if parametric or median and interquartile range if nonparametric. Categorical data were presented as number and percentage. Comparisons between frequencies or percentages were performed by using the chi-square test or the Fisher's exact test. Betweengroup comparisons of continuous variables were performed using the Student's $t$ test or Analysis of Variance for normally distributed variables, and the Mann-Whitney or Kruskal-Wallis tests for nonnormally distributed variables.

Multiple logistic regression analysis, stepwise approach, was used to examine the relationship between serum ferritin, presence and pattern of hepatic iron deposition and presence of NASH or fibrosis. All the variables that were associated with NASH at the univariate analysis with a statistical significance corresponding to a $P$ value up to 0.1 were included in the multivariate analysis. Similarly, logistic regression analysis was performed to find variables associated to advanced fibrosis. A 2-sided $P$ value $<0.05$ was considered significant. All analyses were performed using IBM SPSS (22.0, IBM, New York, USA).

\section{3 | RESULTS}

\subsection{Characteristics of the NAFLD population}

Of the 477 patients initially considered, 2 were subsequently excluded because of suboptimal biopsy sample, 3 because of missing clinical and/or biochemical data and 4 because of compound C282Y/ H63D heterozygosity at the HFE gene test. The demographic, clinical, biochemical and histological details of the 468 patients included in the study are shown in Table 1: the mean age was 47 years, $76 \%$ of patients were of Caucasian ethnicity and $38 \%$ were females. The mean BMI was $30.4 \mathrm{~kg} / \mathrm{m}^{2}$; a history of IFG was observed in $19 \%$ patients and DM in $29 \%$ of patients, of which $65 \%$ were on hypoglycemic agents, mainly metformin. There was a high percentage of patients affected by dyslipidemia (68\%), of which only $25 \%$ were on statin treatment. Hypertension had already been diagnosed in $32 \%$ of subjects, and $62 \%$ were treated mainly with renin-angiotensin system inhibitors. Female subjects were more likely to have diabetes and hypertension ( $45 \%$ vs $29 \%, P<0.001$, and $39 \%$ vs $27 \%$, $P=0.009$, respectively). None of the included patients had radiological or histological evidence of HCC at the time of liver biopsy.

Histological criteria for NASH were fulfilled in 247 (53\%) patients, while advanced fibrosis was prevalent in 89 (19\%) patients ( 81 of which having NASH and 8 with likely 'burnt-out NASH'). The British cohort had a higher prevalence of obesity (mean BMI 31 vs $29, P=0.003$ ), hypertension (37\% vs $24 \%, P=0.003$ ) and dyslipidemia than the Italian cohort ( $77 \%$ vs $55 \%, P<0.0001$ ) and this was reflected by a higher prevalence of patients who fulfilled the criteria for MetS ( $37 \%$ vs $20 \%, P<0.0001$ ). On the other hand, a higher proportion of patients with NASH $(83 \%$ vs $33 \%, P<0.0001)$ and hepatic iron deposition ( $36 \%$ vs $17 \%, P<0.0001)$ was found in the 
TAB LE 1 Demographic, clinical data and laboratory findings of the 468 patients with non-alcoholic fatty liver disease

\begin{tabular}{|c|c|c|c|c|}
\hline & British cohort $(n=282)$ & Italian cohort $(n=186)$ & $P$ & Total $(n=468)$ \\
\hline $\begin{array}{l}\text { Caucasian ethnicity, n } \\
\text { (\%) }\end{array}$ & $168(60)$ & $186(100)$ & $<0.0001$ & $354(76)$ \\
\hline Females, n (\%) & $112(40)$ & $65(35)$ & 0.29 & $177(38)$ \\
\hline $\mathrm{BMI}, \mathrm{kg} / \mathrm{m}^{2}$ & $31 \pm 6$ & $29 \pm 6$ & 0.003 & $30.4 \pm 5.8$ \\
\hline Hypertension, n (\%) & 104 (37) & $45(24)$ & 0.003 & $149(32)$ \\
\hline Dyslipidemia, n (\%) & $217(77)$ & $103(55)$ & $<0.0001$ & $320(68)$ \\
\hline Diabetes, n (\%) & $90(32)$ & $43(23)$ & 0.03 & $133(29)$ \\
\hline IFG, n (\%) & $58(21)$ & $32(17)$ & 0.36 & $90(19)$ \\
\hline MetS, n (\%) & 105 (37) & $38(20)$ & $<0.0001$ & $143(31)$ \\
\hline GGT, U/L & $77(24-130)$ & $66(19-113)$ & 0.009 & $73(24-121)$ \\
\hline Bilirubin, $\mu \mathrm{mol} / \mathrm{L}$ & $11(7-14)$ & $10(7-13)$ & 0.97 & $11(7-14)$ \\
\hline Albumin, g/dL & $4.6(4.4-4.8)$ & $4.6(4.4-4.9)$ & 0.98 & $4.6(4.4-4.8)$ \\
\hline INR > 1.2, n (\%) & $14(5)$ & $1(0.5)$ & 0.0061 & $15(3.3)$ \\
\hline Cholesterol, mmol/L & $5.4(4.5-6.3)$ & $5.1(4.4-5.8)$ & 0.3 & $5.2(4.4-6)$ \\
\hline $\mathrm{HDL}, \mathrm{mmol} / \mathrm{L}$ & $1.2(0.9-1.5)$ & $1.3(1.1-1.5)$ & 0.9 & $1.2(1-1.4)$ \\
\hline $\mathrm{TG}, \mathrm{mmol} / \mathrm{L}$ & $1.8(1-2.4)$ & $1.4(0.9-2)$ & $<0.0001$ & $1.6(1-2.2)$ \\
\hline GLC, $\mathrm{mmol} / \mathrm{L}$ & $5.4(4.4-6.4)$ & $5.2(4.6-5.8)$ & 0.035 & $5.3(4.6-6)$ \\
\hline $\mathrm{F} 2, \mathrm{n}(\%)$ & $21(7)$ & $47(25)$ & $<0.0001$ & $68(15)$ \\
\hline F3, n (\%) & $16(6)$ & $25(13)$ & 0.004 & $41(9)$ \\
\hline F4, n (\%) & $35(12)$ & $13(7)$ & 0.06 & $48(10)$ \\
\hline$\geq F 3, n(\%)$ & $51(18)$ & $38(20)$ & 0.53 & 89 (19) \\
\hline Hepatic iron, n (\%) & 49 (17) & $67(36)$ & $<0.0001$ & $116(25)$ \\
\hline $\mathrm{HC}, \mathrm{n}(\%)$ & $22(8)$ & $20(11)$ & 0.27 & $42(9)$ \\
\hline RES, n (\%) & $14(5)$ & $10(5)$ & 0.84 & $24(5)$ \\
\hline Mixed, n (\%) & $13(5)$ & $37(20)$ & $<0.0001$ & $50(11)$ \\
\hline
\end{tabular}

ALT, alanine aminotransferase; AST, aspartate aminotransferase; BMI, body mass index; GGT, $\gamma$-glutamyl transpeptidase; GLC, serum glucose; HC, hepatocellular iron deposition; HDL, high density lipoprotein; INR, international normalised ratio; NASH, non-alcoholic steatohepatitis; RES, reticuloendothelial system cells iron deposition; TG, triglyceride; TSAT, transferrin saturation.

Data are reported as $\mathrm{n}(\%)$, mean $\pm \mathrm{SD}$, median and interquartile range (IQR).

Italian cohort. No difference in the proportion of patients with advanced fibrosis was found when comparing the 2 cohorts ( $18 \%$ vs $20 \%, P=0.53$ ).

The characteristics of the 247 patients with NASH are shown in Table 2. They were more likely to be older, have a higher BMI and be affected by diabetes when compared to patients without NASH.
Interestingly, the prevalence of MetS was not different between the 2 groups. Diabetic patients with NASH had a higher probability of suboptimal glycemic control if compared to diabetic patients without NASH as shown by a significantly different distribution of HbA1c values (54 (42-96) vs $44(38-55) \mathrm{mmol} / \mathrm{mol}, P=0.02)$, despite a similar proportion of patients on hypoglycemic treatment (data not 
TABLE 2 Comparison of patients according to the presence of NASH

\begin{tabular}{|c|c|c|c|}
\hline & NASH $(n=247)$ & No NASH $(n=221)$ & $P$ \\
\hline British/Italian, n (\%) & $92(37) / 155(63)$ & $190(86) / 31(14)$ & $<0.0001$ \\
\hline Age, y & $50 \pm 13$ & $45 \pm 12$ & 0.014 \\
\hline Females, n (\%) & $99(40)$ & $78(35)$ & 0.29 \\
\hline $\mathrm{BMI}, \mathrm{kg} / \mathrm{m}^{2}$ & $31 \pm 6$ & $29 \pm 6$ & 0.014 \\
\hline Hypertension, n (\%) & $87(35)$ & $62(29)$ & 0.12 \\
\hline Dyslipidemia, n (\%) & $162(66)$ & $158(72)$ & 0.14 \\
\hline Diabetes, n (\%) & $114(46)$ & $49(23)$ & $<0.0001$ \\
\hline IFG, n (\%) & $88(39)$ & $79(40)$ & 0.74 \\
\hline MetS, n (\%) & $81(33)$ & $62(28)$ & 0.26 \\
\hline Alcohol, g/d & $1.3 \pm 4$ & $2.1 \pm 5$ & 0.055 \\
\hline Alcohol > 10 g/d, n (\%) & $11(4.5)$ & $16(7)$ & 0.19 \\
\hline HOMA & $4.1 \pm 3.7$ & $1.7 \pm 1.2$ & 0.001 \\
\hline WCC, $\times 10^{9} / \mathrm{L}$ & $6.56(5.5-7.6)$ & $6.8(5.5-8.1)$ & 0.63 \\
\hline $\mathrm{ALT}, \mathrm{U} / \mathrm{L}$ & $74(43-105)$ & $58(34-72)$ & $<0.0001$ \\
\hline GGT, U/L & $68(25-111)$ & $81(29-133)$ & 0.13 \\
\hline Bilirubin, $\mu \mathrm{mol} / \mathrm{L}$ & $10(7-13)$ & $11(8-14)$ & 0.64 \\
\hline Cholesterol, mmol/l & $5.2(4.5-6)$ & $5.2(4.3-6.1)$ & 0.75 \\
\hline $\mathrm{HDL}, \mathrm{mmol} / \mathrm{L}$ & $1.2(1-1.4)$ & $1.3(1-1.6)$ & 0.15 \\
\hline TG, $\mathrm{mmol} / \mathrm{L}$ & $1.5(0.9-2.1)$ & $1.71 .1-2.3)$ & 0.41 \\
\hline CRP, mg/L & $4.5(0.5-8.5)$ & $3(0.5-5.5)$ & 0.24 \\
\hline Serum Iron, $\mu \mathrm{mol} / \mathrm{L}$ & $17.3(12-22)$ & $18.6(13-23)$ & 0.25 \\
\hline Ferritin, $\mu \mathrm{g} / \mathrm{L}$ & $198(53-343)$ & $181(64-298)$ & 0.42 \\
\hline Ferritin > ULN, n (\%) & $72(26)$ & $50(23)$ & 0.11 \\
\hline TSAT, $\%$ & $24(16-32)$ & $33(24-42)$ & 0.001 \\
\hline Hepatic iron, n (\%) & $66(27)$ & $50(23)$ & 0.31 \\
\hline $\mathrm{HC}$ & $20(8)$ & $22(10)$ & 0.48 \\
\hline RES & $11(4)$ & $13(6)$ & 0.49 \\
\hline Mixed & 35 (14) & $15(7)$ & 0.01 \\
\hline
\end{tabular}

ALT, alanine aminotransferase; BMI, body mass index; CRP, C reactive protein; GGT, $\gamma$-glutamyl transpeptidase; HC, hepatocellular iron deposition; HDL, high density lipoprotein; HOMA IR, homeostatic model for assessment of insulin resistance; RES, reticuloendothelial system cells iron deposition; TG, triglyceride; TSAT, transferrin saturation; WCC, white cells count.

Data are reported as $\mathrm{n}(\%)$, mean $\pm \mathrm{SD}$, median and interquartile range (IQR). shown); also, the HOMA index was higher in NASH patients (4.1 vs $1.7, P=0.001$ ), reflecting higher insulin resistance. Serum transaminases were significantly higher in patients with $\mathrm{NASH}$, with a median value of 1.5-2 $\times$ ULN. When considering general markers of inflammation (CRP), no difference was found between patients with and without NASH.

\subsection{Hepatic iron deposition}

Stainable hepatic iron was found in 116 (25\%) patients: the pattern of iron deposition was mainly $\mathrm{HC}$ in 42 patients (36\%), mainly reticuloendothelial in 24 patients (21\%) and mixed in 50 (43\%) patients. An iron grade 2 was found in 11 (9\%) patients and grade 3 in $3(3 \%)$ patients; none had an iron grade 4 .
Clinical and laboratory data of subjects according to the presence of stainable hepatic iron and to the pattern of iron deposition are shown in Table 3: subjects with stainable hepatic iron were more likely to be male and have a lower BMI, had increasingly higher levels of SF (particularly Mixed $>$ RES $>$ HC, $P<0.0001)$, serum iron $(23.4 \mu \mathrm{mol} / \mathrm{L}$ vs $17 \mu \mathrm{mol} / \mathrm{L}, P<0.0001$ ) and TSAT (37\% vs $27 \%, P=0.04$ ), with a higher proportion of patients with hyperferritinemia ( $60 \%$ vs $15 \%, P<0.0001$ ) and TSAT $>45 \%$ ( $27 \%$ vs $10 \%, P=0.003$, data not shown). In addition, they had significantly higher levels of ALT $(P=0.007)$.

\subsection{SF, iron deposition and liver disease severity}

Hyperferritinemia was found in 122 (26\%) patients, with no significant difference between the 2 cohorts ( $34 \%$ vs $28 \%, P=0.17$ ). 
TAB LE 3 Demographic, clinical data and laboratory findings of the 468 patients with non-alcoholic fatty liver disease according to the presence of stainable iron in the liver and the pattern of iron deposition

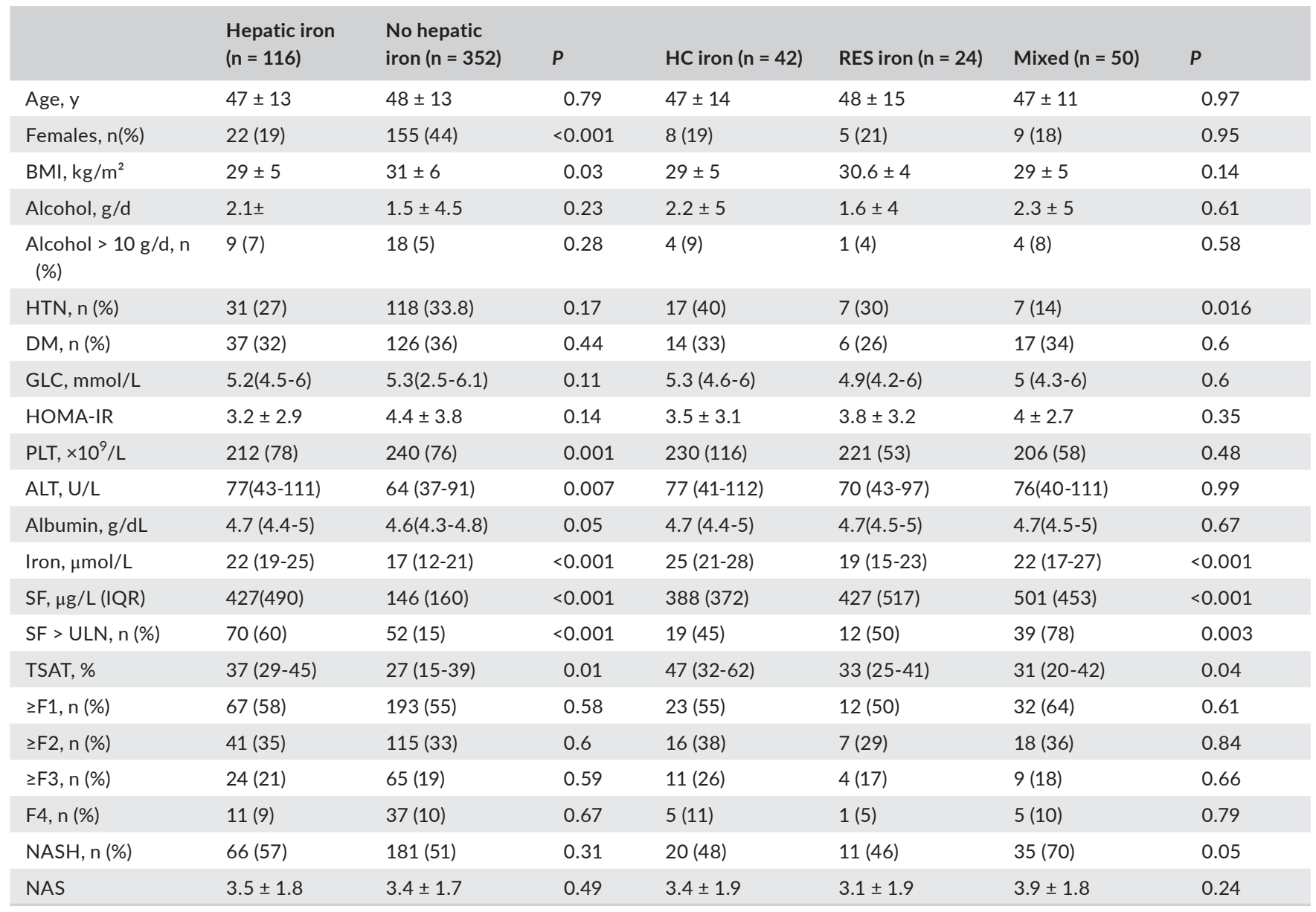

ALT, alanine aminotransferase; BMI, body mass index; DM, type 2 diabetes mellitus; GLC, serum glucose; HC, hepatocellular iron deposition; HOMA IR, homeostatic model for assessment of insulin resistance; HTN, hypertension; NAS, NASH activity score; NASH, non-alcoholic steatohepatitis; PLT, platelets; RES, reticuloendothelial system cells iron deposition; TSAT, transferrin saturation.

Data are reported as $n(\%)$, mean $\pm S D$, median and interquartile range (IQR).

Patients with hyperferritinemia had no difference in sex $(P=0.23)$, ethnicity $(P=0.64)$, cohort (0.17), age (0.39), BMI $(P=0.83)$, prevalence of diabetes and or/IFG compared to non hyperferritinemics. When looking at mild alcohol consumption, an increasing trend in SF values was found for increasing alcohol intake; however, the prevalence of hyperferritinemia was not different between abstinent vs mild drinkers $(P=0.1)$, even when considering subclasses of alcohol intake ( $P=0.097$, Table SS).

Looking at the single components of histological NASH, a positive association was found between SF and increasing degree of steatosis $(P=0.008)$ but not with hepatocellular ballooning or lobular inflammation and overall SF did not associate with diagnosis of NASH (Table 2). Interestingly, SF showed a peculiar pattern throughout fibrosis stages, increasing from F0-F1 to F3, and subsequently decreasing in cirrhosis (Figure 1). When comparing the inter-group SF distribution, it was significantly higher for F3 compared to F0-F1 $(P=0.024)$, with an average increase of $14 \%$ from F0-F1 to F2 and of 61\% from F2 to F3 and a decrease of $43 \%$ from $\mathrm{F} 3$ to F4.
On the other hand, patients with a mixed pattern of iron deposition were more likely to have NASH if compared to patients with other patterns of iron deposition or to patients without hepatic iron. No difference was seen between patients with and without hepatic stainable iron when considering the individual components of histological NASH, NAS $>3$ or $>5$ (data not shown).

No association was found between presence of stainable hepatic iron and fibrosis (mild, significant, advanced).

As shown in Table 4, at the multivariate analysis, BMI (OR 1.06, 95\% Cl 1.02-1.11, $P=0.005$ ), presence of diabetes (OR 2.13, 95\% Cl 1.31-3.45, $P=0.01$ ), ALT levels (OR 1.012, 95\% Cl 1.006-1.017, $P<0.0001)$ and a mixed pattern of hepatic iron deposition, (OR 2.23, 95\% Cl 1.08-4.6, $P=0.03$ ) were independently associated with the presence of NASH. Such variables remained the only significantly associated even after removing patients with TSAT higher than $45 \%$ and correcting for cohort and sex or when including alcohol as a categorical or continuous variable but not when considering each cohort separately (data not shown). 


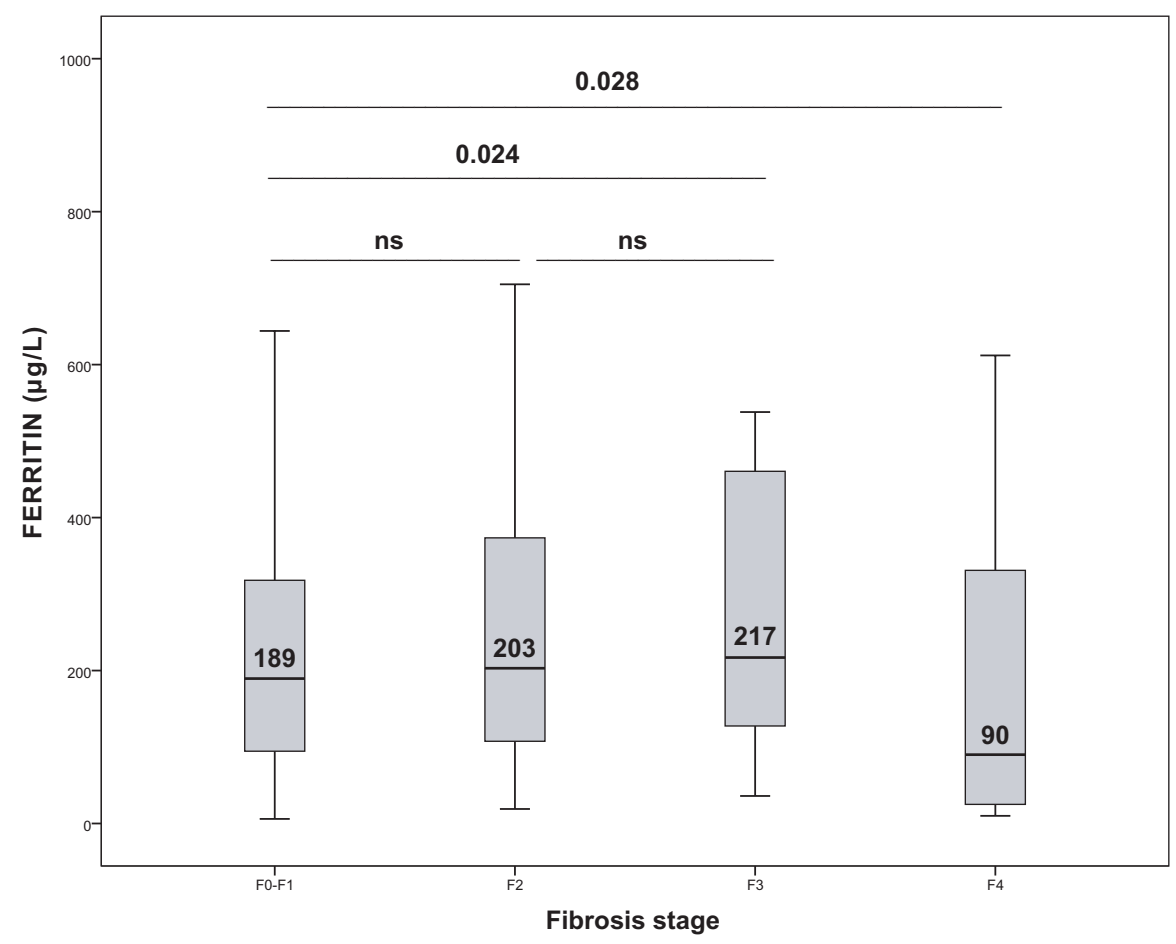

FIGURE 1 Serum ferritin according to fibrosis stage, comparison between groups

TABLE 4 Predictors of NASH at univariate and multivariable analysis

\begin{tabular}{|lllll|} 
& OR $(95 \% \mathrm{Cl})$ & $P$ & OR $(95 \% \mathrm{Cl})$ & $P$ \\
\hline Age & $1.01(1.003-1.03)$ & 0.01 & & $\mathrm{~ns}$ \\
\hline Sex & $1.22(0.84-1.78)$ & 0.28 & & $\mathrm{~ns}$ \\
\hline BMI & $1.06(1.02-1.1)$ & 0.002 & $1.06(1.02-1.11)$ & 0.005 \\
\hline Hypertension & $1.4(0.92-2.01)$ & 0.1 & & $\mathrm{~ns}$ \\
\hline Diabetes & $2.11(1.39-3.21)$ & $<0.0001$ & $2.13(1.31-3.45)$ & 0.01 \\
\hline ALT & $1.007(1.003-1.01)$ & $<0.0001$ & $1.01(1.006-1.017)$ & $<0.0001$ \\
\hline Ferritin & $1.001(1-1.001)$ & 0.07 & & $\mathrm{~ns}$ \\
\hline Iron pattern & & & & 0.43 \\
\hline \multicolumn{1}{l}{ HC iron } & $0.86(0.45-1.63)$ & 0.64 & $0.72(0.32-1.63)$ & 0.12 \\
\hline RES iron & $0.79(0.35-1.83)$ & 0.59 & $0.64(0.24-1.72)$ & 0.03 \\
\hline
\end{tabular}

$\mathrm{ALT}$, alanine aminotransferase; $\mathrm{BMI}$, body mass index; $\mathrm{Cl}$, confidence interval; $\mathrm{HC}$, hepatocellular iron deposition; OR, odds ratio; RES, reticuloendothelial system cells iron deposition.
Advanced fibrosis was associated with advanced age (OR 1.04, $95 \% \mathrm{Cl} 1.01-1.06, P=0.01$ ), presence of diabetes (OR 3.09, $95 \% \mathrm{Cl}$ $1.65-5.79, P<0.0001)$ and low platelet count (OR 0.987; $95 \% \mathrm{Cl}$ $0.982-0.992, P<0.0001)$ but not with serum ferritin.

\section{4 | DISCUSSION}

In this study, we showed that a mixed pattern of hepatic iron deposition is associated with the presence of NASH, while ferritin levels increase with worsening of fibrosis up to a pre-cirrhotic stage but are not independent predictors of advanced fibrosis.
Stainable hepatic iron was found in $25 \%$ of patients, in line with previous studies reporting a prevalence ranging from $14 \%$ to $50 \% .{ }^{23-25}$ The degree of iron accumulation was rarely above a mild to moderate degree and never above grade 3 : this can be considered a typical characteristic of NAFLD patients with stainable hepatic iron, and would be one of the reasons explaining why many studies of phlebotomy in NAFLD have failed to show a benefit or improvement in liver histology. ${ }^{26,27}$

A mixed pattern of iron deposition was the most common, accounting for $43 \%$ of patients with stainable hepatic iron and this is consistent with data from the literature. ${ }^{5,23,24}$ Noteworthy, a mixed pattern of iron deposition was independently associated to the 
presence of histological NASH. However, hepatic iron was not associated with fibrosis, which was recently identified as the main determinant of prognosis in NAFLD patients. This would suggest that NAFLD patients who tend to accumulate iron in both hepatocytes and macrophages for multiple factors (genetics, local effect of inflammation on iron metabolism, leaking from dying hepatocytes and subsequent phagocytosis by liver macrophages ${ }^{28}$ ) are at higher risk to have a more active form of liver disease, but might still require a further hit to trigger and maintain fibrogenesis, as per the 'multiple hit' hypothesis. $^{3}$

Our results are different from those previously presented by other groups. In 849 patients enrolled in the NASH CRN, ${ }^{24}$ despite similar rates of hepatic iron and mixed pattern deposition, it was only RES iron which was associated with advanced fibrosis. At variance to these data, in another study by Valenti et al ${ }^{23}$ including 587 NAFLD patients, hepatocellular iron was associated with a 1.7-fold higher risk of fibrosis $\geq \mathrm{F} 1$.

These discrepancies are difficult to explain. Preliminary data from next generation sequencing studies have shown how variants/ polymorphisms of genes involved in iron metabolism, other than HFE, could have an impact on SF levels and hepatic iron in NAFLD patients, ${ }^{29,30}$ therefore differences linked to ethnicity and geographical provenience are to be expected.

As regards to SF, the prevalence of hyperferritinemia was $26 \%$ in our cohort. SF was higher in patients with detectable hepatic iron, particularly those with a mixed pattern of iron deposition. Similarly, in a study by Ryan et al, ${ }^{31} \mathrm{SF}$ correlated with liver iron determined by MRI in 51 NAFLD patients, and with iron deposition in RES in a retrospective cohort of 404 NAFLD patients.

Interestingly, in our study SF was associated with increasing degree of steatosis but not with NASH and, more importantly, SF was not independently associated with fibrosis. Previous studies have indeed tried to understand the meaning of a raised SF in NAFLD patients, particularly examining the role of SF as a noninvasive marker of NASH and/or fibrosis, some underlying its positive correlation with increasing fibrosis, ${ }^{6,7}$ others obtaining opposite results. ${ }^{8}$ In a study including 1201 patients with biopsy-proven NAFLD, SF was associated with presence of steatosis, lobular inflammation and ballooning, but had suboptimal diagnostic performance for detecting significant or advanced fibrosis. ${ }^{32}$

When addressing the potential effect of mild-moderate alcohol intake on SF, a previous observational study on more than 12000 patients showed that consumption of 2 or more alcoholic drinks per day was associated with significant risk of iron overload, whereas consumption of a lesser amount was associated with reduced risk of iron deficiency. ${ }^{33}$ In our cohort, SF increased with increasing degree of alcohol intake, but only patients who drank more than $10 \mathrm{~g} /$ day of alcohol showed significantly higher SF when compared to total abstinent and lower alcohol intake subgroups. Moreover, there was no association between alcohol intake and hepatic iron deposition, NASH or fibrosis, suggesting that in NAFLD patients, the effect of mild alcohol consumption on iron metabolism and NASH is negligible, and different pathways are involved.
Few studies have explored the role of diet and its influence on SF and iron levels: although there are not many solid data, the consumption of red meat or insufficient amount of vegetables, common in MetS patients, seems to be associated with higher SF levels. ${ }^{34}$ Unfortunately, we did not have such information from our cohort.

Considering our results, and the fact that ferritin levels in NAFLD patients tend to be higher than in haemochromatosis patients with the same amount of hepatic iron, ${ }^{35}$ hyperferritinemia in NAFLD might be due both to increased iron stores (without excluding the direct release by macrophage/hepatocytes in response to necrosis and inflammation-mediated mechanisms) and to the systemic inflammatory and metabolically altered status typical for these patients rather than reflect hepatic disease severity. This would be true in our NAFLD population even if raised SF was not associated with high CRP (a suboptimal marker of inflammatory activity) or single MetS components. Moreover, this would explain the reduction of SF seen in cirrhotic patients, where the extinguishing inflammation, the decrease in liver synthetic activity and the development of portal hypertension, could concur in reducing iron adsorption and ferritin synthesis.

The strengths of this study are as follows: first, the number of included patients and the multicentric structure (allowing inclusion of patients from Northern and Southern Europe); secondly, the precise histological review, performed by 1 dedicated histopathologist in each centre using pre-determined, widely used criteria.

The limitations are linked to the retrospective type of the study, therefore some missing clinical data, with a possible underestimation of MetS, the lack of HFE genetic analysis in patients with SF within the range of normal, and the lack of quantitative measurement of hepatic iron concentration ( $\mathrm{HIC}$ ) and cytokines, which would have permitted a more accurate description of the hepatic iron content and inflammatory status of our patients.

In conclusion, the findings presented here allow us to identify 2 possible 'iron signatures': (a) in NAFLD patients SF is frequently high, but in most cases iron does not accumulate in the liver, therefore SF likely reflects a systemic dysmetabolic/inflammatory state; (b) a minority of NAFLD patients with high SF display hepatic iron accumulation in RES cells and hepatocytes (mixed pattern) and have NASH: here hepatic iron accumulation likely results from more pronounced intrahepatic necro-inflammatory events leading to an 'iron retention phenomenon' possibly driven by hepcidin induction.

Do increased iron and ferritin have a direct causative role in $\mathrm{NASH}$ ? Unfortunately, answering such an important question was beyond the aim of the study; still, an hypothesis can be made: the damaging and pro-inflammatory activity of hepatocellular iron has been already established by a number of studies (with a less clear role of RES iron) ${ }^{36}$; interestingly, also ferritin has been reported to act as a proinflammatory cytokine able to activate hepatic stellate cells involved in liver fibrosis. ${ }^{37}$

These hypotheses need corroboration; however, we think that our results provide a further confirmation of the complex relationship between NAFLD and iron metabolism, likely regulated by 
different and antagonistic pathways: this is intriguing and deserves to be explored by future studies.

\section{ACKNOWLEDGEMENTS}

E.B. was supported by a fellowship-grant from AIGO-M.I.M.I. (Associazione Italiana Gastroenterologi Ospedalieri-Montecatini Interactive Medicine International); A.P. was recipient of a Telethon grant (\#GGP14285)

\section{CONFLICTS OF INTEREST}

The authors do not have any disclosures to report.

\section{ORCID}

Elena Buzzetti (iD https://orcid.org/0000-0002-4462-7935

Salvatore Petta (iD https://orcid.org/0000-0002-0822-9673

Roberta Manuguerra iD https://orcid.org/0000-0002-5446-8229

Tu Vinh Luong (iD https://orcid.org/0000-0002-7598-7319

Daniela Cabibi iD https://orcid.org/0000-0001-6571-6577

Elena Corradini (iD https://orcid.org/0000-0001-9477-2164

Antonio Craxi (iD https://orcid.org/0000-0002-4480-9544

Massimo Pinzani iD https://orcid.org/0000-0003-1398-1541

Emmanuel Tsochatzis iD https://orcid.org/0000-0001-5069-2461

Antonello Pietrangelo (iD https://orcid.org/0000-0002-7411-935X

\section{REFERENCES}

1. Ratziu V, Bellentani S, Cortez-Pinto H, Day C, Marchesini G. A position statement on NAFLD/NASH based on the EASL 2009 special conference. J Hepatol. 2010;53(2):372-384.

2. Younossi ZM, Stepanova M, Negro F, et al. Nonalcoholic fatty liver disease in lean individuals in the United States. Medicine (Baltimore). 2012;91(6):319-327.

3. Buzzetti E, Pinzani M, Tsochatzis EA. The multiple-hit pathogenesis of non-alcoholic fatty liver disease (NAFLD). Metabolism. 2016;65(8):1038-1048.

4. Trombini P, Piperno A. Ferritin, metabolic syndrome and NAFLD: elective attractions and dangerous liaisons. J Hepatol. 2007;46(4):549-552.

5. Ryan JD, Armitage AE, Cobbold JF, et al. Hepatic iron is the major determinant of serum ferritin in NAFLD patients. Liver Int. 2018;38(1):164-173.

6. Manousou P, Kalambokis G, Grillo F, et al. Serum ferritin is a discriminant marker for both fibrosis and inflammation in histologically proven non-alcoholic fatty liver disease patients. Liver Int. 2011;31(5):730-739.

7. Kowdley KV, Belt P, Wilson LA, et al. Serum ferritin is an independent predictor of histologic severity and advanced fibrosis in patients with nonalcoholic fatty liver disease. Hepatology. 2012;55(1):77-85

8. Angulo P, George J, Day CP, et al. Serum ferritin levels lack diagnostic accuracy for liver fibrosis in patients with nonalcoholic fatty liver disease. Clin Gastroenterol Hepatol. 2014;12(7):1163-1169. e1161.

9. Buzzetti E, Lombardi R, De Luca L, Tsochatzis EA. Noninvasive assessment of fibrosis in patients with nonalcoholic fatty liver disease. Int J Endocrinol. 2015;2015:343828.

10. Hagstrom $\mathrm{H}, \mathrm{Nasr} \mathrm{P}$, Bottai $\mathrm{M}$, et al. Elevated serum ferritin is associated with increased mortality in non-alcoholic fatty liver disease after 16 years of follow-up. Liver Int. 2016;36(11):1688-1695.

11. Pietrangelo A. Iron and the liver. Liver Int. 2016;36(Suppl 1):116-123.

12. Ahmed U, Latham PS, Oates PS. Interactions between hepatic iron and lipid metabolism with possible relevance to steatohepatitis. World J Gastroenterol. 2012;18(34):4651-4658.

13. Deugnier Y, Turlin B. Pathology of hepatic iron overload. Semin Liver Dis. 2011;31(3):260-271.

14. European Association for the Study of the Liver (EASL), European Association for the Study of Diabetes (EASD), European Association for the Study of Obesity (EASO). EASL-EASD-EASO Clinical Practice Guidelines for the management of non-alcoholic fatty liver disease. J Hepatol. 2016;64(6):1388-1402.

15. Chatrath H, Vuppalanchi R, Chalasani N. Dyslipidemia in patients with nonalcoholic fatty liver disease. Semin Liver Dis. 2012;32(1):22-29.

16. National Cholesterol Education Program (NCEP) Expert Panel on Detection, Evaluation, and Treatment of High Blood Cholesterol in Adults (Adult Treatment Panel III). Third Report of the National Cholesterol Education Program (NCEP) Expert Panel on Detection, Evaluation, and Treatment of High Blood Cholesterol in Adults (Adult Treatment Panel III) final report. Circulation. 2002;106(25):3143-3421.

17. Brunt EM. Histopathology of non-alcoholic fatty liver disease. Clin Liver Dis. 2009;13(4):533-544.

18. Sanyal AJ, Brunt EM, Kleiner DE, et al. Endpoints and clinical trial design for nonalcoholic steatohepatitis. Hepatology. 2011;54(1):344-353.

19. Kleiner DE, Brunt EM, Van Natta M, et al. Design and validation of a histological scoring system for nonalcoholic fatty liver disease. Hepatology. 2005;41(6):1313-1321.

20. Angulo $\mathrm{P}, \mathrm{Kleiner} \mathrm{DE}, \mathrm{Dam}$-Larsen $\mathrm{S}$, et al. Liver fibrosis, but no other histologic features, is associated with long-term outcomes of patients with nonalcoholic fatty liver disease. Gastroenterology. 2015;149(2):389-397.e310.

21. Ekstedt $\mathrm{M}$, Hagstrom $\mathrm{H}, \mathrm{Nasr} \mathrm{P}$, et al. Fibrosis stage is the strongest predictor for disease-specific mortality in NAFLD after up to 33 years of follow-up. Hepatology. 2015;61(5):1547-1554.

22. Turlin B, Deugnier Y. Evaluation and interpretation of iron in the liver. Semin Diagn Pathol. 1998;15(4):237-245.

23. Valenti L, Fracanzani AL, Bugianesi E, et al. HFE genotype, parenchymal iron accumulation, and liver fibrosis in patients with nonalcoholic fatty liver disease. Gastroenterology. 2010;138(3): 905-912.

24. Nelson JE, Wilson L, Brunt EM, et al. Relationship between the pattern of hepatic iron deposition and histological severity in nonalcoholic fatty liver disease. Hepatology. 2011;53(2):448-457.

25. Maliken BD, Nelson JE, Klintworth HM, Beauchamp M, Yeh MM, Kowdley KV. Hepatic reticuloendothelial system cell iron deposition is associated with increased apoptosis in nonalcoholic fatty liver disease. Hepatology. 2013;57(5):1806-1813.

26. Adams LA, Crawford DH, Stuart K, et al. The impact of phlebotomy in nonalcoholic fatty liver disease: a prospective, randomized, controlled trial. Hepatology. 2015;61(5):1555-1564.

27. Laine F, Ruivard M, Loustaud-Ratti V, et al. Metabolic and hepatic effects of bloodletting in dysmetabolic iron overload syndrome: a randomized controlled study in 274 patients. Hepatology. 2017;65(2):465-474. 
28. Corradini E, Pietrangelo A. Iron and steatohepatitis. J Gastroenterol Hepatol. 2012;27(suppl 2):42-46.

29. Corradini E, Bernardis I, Dongiovanni P, et al. Rare ceruloplasmin variants are associated with hyperferritinemia and increased hepatic iron in NAFLD patients: results from a NGS study. The International Liver Congress ILC, Paris, 2018.

30. Valenti L, Rametta R, Dongiovanni P, et al. The A736V TMPRSS6 polymorphism influences hepatic iron overload in nonalcoholic fatty liver disease. PLoS ONE. 2012;7(11):e48804.

31. Ryan JD, Armitage AE, Cobbold JF, et al. Hepatic iron is the major determinant of serum ferritin in NAFLD patients. Liver Int. 2018;38:164-173.

32. Yoneda $M$, Thomas $E$, Sumida $Y$, et al. Clinical usage of serum ferritin to assess liver fibrosis in patients with non-alcoholic fatty liver disease: proceed with caution. Hepatol Res. 2014;44(14):E499 -E502.

33. loannou GN, Dominitz JA, Weiss NS, Heagerty PJ, Kowdley KV. The effect of alcohol consumption on the prevalence of iron overload, iron deficiency, and iron deficiency anemia. Gastroenterology. 2004;126(5):1293-1301.

34. Avila F, Echeverria G, Perez D, et al. Serum ferritin is associated with metabolic syndrome and red meat consumption. Oxid Med Cell Longev. 2015;2015:769739.

35. Nelson JE, Brunt EM, Kowdley KV; Nonalcoholic Steatohepatitis Clinical Research Network. Lower serum hepcidin and greater parenchymal iron in nonalcoholic fatty liver disease patients with C282Y HFE mutations. Hepatology. 2012;56(5):1730-1740.
36. Handa P, Vemulakonda AL, Maliken BD, et al. Differences in hepatic expression of iron, inflammation and stress-related genes in patients with nonalcoholic steatohepatitis. Ann Hepatol. 2017;16(1):77-85.

37. Ruddell RG, Hoang-Le D, Barwood JM, et al. Ferritin functions as a proinflammatory cytokine via iron-independent protein kinase $C$ zeta/nuclear factor kappaB-regulated signaling in rat hepatic stellate cells. Hepatology. 2009;49(3):887-900.

\section{SUPPORTING INFORMATION}

Additional supporting information may be found online in the Supporting Information section at the end of the article.

How to cite this article: Buzzetti E, Petta S, Manuguerra R, et al. Evaluating the association of serum ferritin and hepatic iron with disease severity in non-alcoholic fatty liver disease. Liver Int. 2019;39:1325-1334. https://doi.org/10.1111/ liv.14096 